6

\title{
Binaries in Globular Clusters
}




\title{
DYNAMICS AND BINARY (TRANS)FORMATION
}

\section{IN GLOBULAR CLUSTERS}

\author{
PIET HUT \\ Institute for Advanced Study \\ Princeton, NJ 08540, U.S.A.
}

\begin{abstract}
Globular clusters form ideal laboratories for studying the interactions between stellar evolution and stellar dynamics. In the past, highly exceptional systems such as X-ray binaries and later millisecond pulsars have provided us with useful diagnostic tools. However, the fate of the bulk of the more normal stars has remained less clear. At present, rapid progress is being made in our understanding of the distributions of normal stars and primordial binaries, as well as their most abundant reaction products: blue stragglers and binaries that are produced through exchange encounters with other single stars or binaries. The complexity of the network of exchange reactions is illustrated through some specific examples, such as a formation scenario for the hierarchical triple system containing the millisecond pulsar PSR B1620-26 in M4, the first triple star system ever detected in a globular cluster.
\end{abstract}

\section{Introduction}

During the last decade, a wealth of observational data has been obtained that can help us understand the interactions between stars in dense stellar systems. For example, in globular clusters a large number of millisecond pulsars have been detected, new low-luminosity X-ray sources have been found, and many blue stragglers have been discovered in the inner regions of some of the densest clusters. All these objects suggest a complex history of interactions between single stars and/or binary stars, due to close encounters or even collisions.

Fortunately, during the last decade similar progress has been made in the modeling of dense star clusters. The mechanism of core collapse has 
become better understood, and the evolution of a star cluster past core collapse has been modeled in considerable detail, using a variety of modeling approaches. The recent discovery that globular clusters contain a significant binary population has further complicated these attempts of cluster modeling. Since the binding energy of all these binaries form a very important factor in the overall energy budget of a cluster, care has to be taken in following the changes in their internal orbital parameters. In turn, to determine these changes, some of the dominant effects of stellar evolution have to be taken into account as well.

Many references to previous work in this general area can be found in a recent review of observational as well as theoretical progress in our understanding of binaries in globular clusters (Hut et al. 1992).

\section{Dynamical Transformations of Primordial Binaries}

Binaries play a central role in cluster dynamics. If their orbital speed exceeds that of the velocity dispersion of the single stars, the tendency toward energy equipartition during encounters will transfer some of the internal kinetic energy to passing stars. Doing so, energy conservation causes them to shrink, while the negative heat capacity of self-gravitating systems causes them to heat up further, to higher orbital speeds (Lynden-Bell's 'donkey effect': trying to slow down particles in a Kepler orbit speeds them up, and vice versa).

The 'gravitational fusion' of single stars into double stars is thus one mechanism that can heat a cluster, in order to balance the energy losses due to the evaporation of stars and the heat flow through the cluster toward the colder halo. Other mechanisms can play a role as well in fueling the central heat engine needed to balance the heat flow from the core to the cluster halo. Mass loss through stellar evolution (especially the much more rapid stellar evolution of the relatively heavier merger remnants) can indirectly heat a cluster through the paradoxical effect of carrying off kinetic energy simply because the potential energy carried off per unit mass is much larger, and tilts the balance towards an effective heating. Similarly, the formation of a modest black hole can also cause a heating of the cluster, through the selective disruption of stars on low-energy orbits near the hole (for both mechanisms, see the review by Goodman 1992).

It is far from clear to what extent these various heating mechanisms compete with each other in actual globular cluster cores. Order-of-magnitude estimates indicate that they all can be significant, depending on the precise conditions in the cores, as well as on the nature of the stars. For example, white dwarfs, neutron stars and stellar-mass black holes are likely to produce energy by dynamical binary formation and hardening, while 
main-sequence stars and giants are likely to suffer physical collisions while attempting to do so.

Whatever the detailed mix of energy sources in individual clusters may turn out to be, it is becoming clear that binaries are crucially important to the energetics of globular clusters. For example, observations of primordial binaries in globular clusters indicate that the binary abundance in globular clusters is not much smaller than that in the Galactic disk and halo (cf., Hut et al. 1992). This suggests that $\gtrsim 10 \%$ of the stellar objects in a cluster may be binaries with an orbit of $\lesssim 1 \mathrm{AU}$, which implies an average binding energy per binary of $\gtrsim 10$ times that of the average kinetic energy of single cluster stars. This simple reasoning leads to the remarkable conclusion that the internal energy reservoir in binary binding energies may well exceed the total amount of kinetic energy in the cluster as a whole (in the form of center-of-mass motion of single stars and binaries).

With binary stars having locked up the bulk of the energy content of a typical globular cluster, we cannot afford to neglect the transformations in binary properties that take place during the course of normal stellar evolution. The reason is that stellar encounters do not have a monopoly on changing the energy and angular momentum of binaries; isolated binaries, too, have plenty of ways of changing their appearance in complicated ways (cf., Pols \& Marinus 1993).

Even a partial list of some of the processes involved in isolated binary evolution gives an idea of the complexity of the physics: tidal capture, magnetic braking, gravitational radiation, runaway mass transfer, and common envelope evolution. Take into account the manifold perturbations and disruptions that can occur during interactions with passing stars or binaries, and the full complexity of the problem becomes clear. The feedback mechanisms between stellar dynamics and stellar evolution in globular clusters play a vital role in the evolution of the cluster as a whole. The term 'ecology', used by Douglas Heggie in his recent 'News and Views' article in Nature (Heggie 1992), indeed captures the essence of this interplay.

\section{Fully Automatized Three-Body Scattering Experiments}

The first set of binary-single-star scattering experiments were reported by Hills (1975). In these experiments, most encounters took place at zero impact parameter. The first direct determination of accurate cross sections and reaction rates for binary-single-star scattering was made by Hut \& Bahcall (1983). For each type of total or differential cross section, a detailed search of impact parameter space was performed as a pilot study, before production runs were started. The problem with the choice of impact parameter (lateral offset from a head-on collision, as measured at infinity) is 
this: allowing too large an impact parameter can imply a large waste of computer time on uninteresting orbits; while choosing too small an impact parameter will yield a systematic underestimate of some cross sections, since some encounters of interest will be missed.

The first automatic determination of cross sections and reaction rates for binary-single-star scattering has been performed by Hut \& McMillan (1994). Rather than relying on human inspection of pilot calculations, their software package includes an automatic feedback system that ensures nearoptimal coverage of parameter space while guaranteeing completeness. The basic idea is to maintain a safety zone outside the 'bull's eye' region, defined as the area where 'interesting' reactions take place (where 'interesting' is defined according to the particular interest underlying a particular set of experiments). Allowing for the possibility of dynamically enlarging this safety zone guarantees rapid convergence towards accurate cross sections. One of our first applications has been to determine exchange cross sections for the general problem of binary-single-star scattering with unequal masses (Heggie, Hut \& McMillan 1994). Another application is concerned with the formation of triple systems in binary-binary encounters, in the limit where the tightest of the two binaries can be approximated as a single point mass (Rasio, McMillan \& Hut 1995), as will be discussed in the next section. References to earlier papers on 3-body scattering can be found in the recent papers by Hills (1992), Heggie \& Hut (1993), Hut (1993a), Sigurdsson \& Phinney (1993).

The software, developed by Hut \& McMillan (1994) to perform the automated scattering calculations, was designed in a multi-layer objectoriented approach and is implemented in $\mathrm{C}++$. At the lowest layer, the orbit integration engine consists of a fourth-order variable-time-step Hermite integrator. What is novel here is the time step criterion used: through onestep iteration, time symmetry is guaranteed to very high accuracy (Hut, Makino \& McMillan 1994), which leads to spectacular improvements in long-term stability of the integration scheme, something that is essential for the treatment of long-lasting resonance scattering events.

On top of this lowest layer, there are several layers that contain: 1) checks to determine whether a given scattering experiment has reached its final outgoing state; 2 ) checks to allow optimization features to be activated, such as analytical integration of inner and outer orbits of hierarchical triple systems in which the outer orbital period vastly exceeds the inner orbital period; 3) diagnostic functions to store information describing the build-up of energy errors; 4) various bookkeeping functions that chart the overall character of the orbits (i.e., democratic vs. hierarchical resonance states); 5 ) checks for overlap of stellar radii, in which case merging routines are invoked that can replace two colliding stars with a single merger product. 
On top of these layers, the first user-accessible layer contains a singlescattering command, with a large number of options. The masses and radii of the stars can be specified, as well as the orbital parameters of the binary, the impact parameter of the encounter, and the relative velocity, asymptotically far before the encounter. The initial distance from which the integration starts is determined automatically (and will be much larger for, say, a $10 \mathrm{M}_{\odot}$ black hole approaching a given binary compared to a $0.5 \mathrm{M}_{\odot}$ dwarf). In addition, an overall accuracy parameter gives a handle on the cost/performance tradeoff. In practice, typical relative energy errors can be easily kept as small as $10^{-10}$; our production runs usually aim at errors of order $10^{-6}$, allowing a speed-up of a factor ten in computer time with respect to the most accurate integrations attainable.

The next layer contains all the management software to conduct a series of scattering experiments. Depending on the type of total or differential cross section requested, the user can choose an appropriate command to activate a 'beam' of single stars aimed at the 'target plate' of binary stars. After a few minutes, a preliminary report appears on the screen, with estimates of all relevant cross sections plus their corresponding error bars. Thereafter, subsequent reports appear, each one after a four times longer interval. Because of the Monte Carlo nature of the orbit parameter sampling, each following report carries error bars that are half the size of the corresponding ones in the previous report. Thus reasonable estimates can often be obtained in ten or fifteen minutes, with more accurate results following in an hour (on a fast modern work station).

Another higher-level layer, on top of the cross section manager, is the Maxwellian rate estimator. After specification of the stellar characteristics, the binary orbit, and the velocity dispersion of the single stars, this estimator computes cross sections at different points under the Maxwellian velocity distribution curve, multiplying the results by the Maxwellian weight factor, and adding those to obtain reaction rates. As before, the longer one is willing to wait, the more accurate the rates become (through an automatic increase in the number of velocity points as well as an continued increase in accuracy of each of cross sections determined at each velocity points).

Further still-higher-level layers can be added with very little additional time investment. With the complexity of orbit integration and scattering management hidden in the various modules, it is relatively straightforward to implement additional levels. For example, one could easily address the inverse scattering problem: given a final system, what is the relative probability that such a system originated from different initials conditions? This could be solved as follows: after specifying the velocity dispersion and other stellar parameters, an automatic tabulation of Maxwellian reaction rates 
could be performed, while filtering the results to allow only those scattering experiments to be counted that lead to the desired range of final parameters.

Currently, several projects are underway in which we are applying our scattering package to various problems. The initial results are very encouraging, in two respects. First, it has become far easier to carry out many long production runs in so-far unexplored parameter ranges. Second, our package invites real-time experimentation in these new parameter ranges. Not only are new cross sections available in minutes, there are also hooks at all levels to allow graphic output. Actually watching individual resonance scattering orbits in real time has already showed us, for example, that there is a need for an extension of the present classification: apart from the traditional division in democratic and hierarchical resonances, sufficient to cover equal-mass scattering, there are other types that do not fit in these two categories. An example is the class of 'flip-flop' resonances, in which one lighter star is being exchanged several times between repeated encounters of two heavier stars (Heggie, Hut \& McMillan 1994).

There are two ingredients that have made these new developments possible. One is the increase in available computer speed of a factor of a thousand, from the earliest scattering experiments performed by Hills (1975) to the ones reported by Hut \& Bahcall (1983), with a similar increase of a factor of a thousand between then and now. The second ingredient is the wider availability at present of higher-level computer languages that enable and invite a more flexible and modular approach to programming, making automation far easier.

\section{Formation of the PSR B1620-26 Triple System in M4}

The hierarchical triple system containing the millisecond pulsar PSR B162026 in M4 is the first triple star system ever detected in a globular cluster (Backer, Foster \& Sallmen 1993; Thorsett, Arzoumanian \& Taylor 1993). Such systems should form in globular clusters as a result of dynamical interactions between binaries (Mikkola 1984; Hut 1992). Specifically, Rasio, McMillan \& Hut (1995) have proposed that the triple system containing PSR B1620-26 formed through an exchange interaction between a wide primordial binary and a pre-existing binary millisecond pulsar (BMP).

This scenario has the advantage of reconciling the $\sim 10^{9} \mathrm{yr}$ timing age of the pulsar with the much shorter lifetime of the triple system in the core of M4. Using the automated three-body scattering package, we calculated a large number of interactions between the BMP and a primordial binary to determine the triple-formation cross section as well as the predicted characteristics of the triple systems. Some results are shown in Fig. 1. 


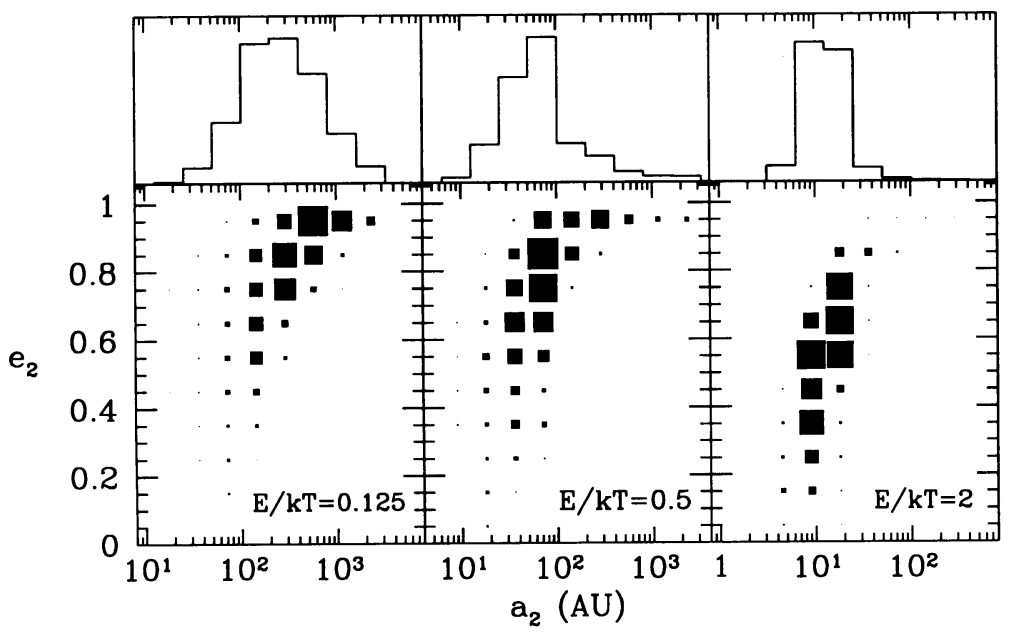

Figure 1. Distribution of the semi-major axis $a_{2}$ of the triple's outer orbit (above) and distribution in the $\left(a_{2}, e_{2}\right)$ plane (below). The size of each square dot is proportional to the differential cross-section for producing a triple system with specific orbital parameters. The incoming primordial binary contained two $0.5 \mathrm{M}_{\odot}$ stars with semi-major axis $a_{\mathrm{MS}} \simeq 50 \mathrm{AU}$ (left), $13 \mathrm{AU}$ (middle), and $3 \mathrm{AU}$ (right).

Since the eccentricity of the inner BMP in the presently observed system is very small $\left(e_{1} \approx 0.025\right)$, the BMP cannot have been perturbed very much during the exchange interaction. This places a limit on how close either of the two MS stars in the primordial binary can have approached the BMP. In the calculations of exchange interactions, the BMP was treated as a single mass and given an effective radius $R_{\mathrm{e}}$ such that close passages with periastron separation $r_{\mathrm{p}}>R_{\mathrm{e}}$ cannot produce an eccentricity larger than observed today.

Calculations of the induced eccentricity in binary-single interactions, also performed in the Starlab environment (Hut 1993b) in which the scattering package was written, indicate that $R_{\mathrm{e}} \approx 3 a_{1} \simeq 2.4 \mathrm{AU}$ for this system, where $a_{1}$ is the semi-major axis of the BMP. Fig. 1 shows the probability distributions of $a_{2}$ and $e_{2}$, the semi-major axis and eccentricity of the outer orbit in the triple system for several typical cases. For very soft incoming binaries (much wider than the BMP), the outer orbit tends to be very eccentric (marginally bound), with a wide distribution of separations extending over more than a factor 10 (cf., $E=0.125$; here $E$ is the binding energy of the incoming primordial binary in units of " $k T$ " in the cluster).

For harder incoming binaries, the average eccentricity gets smaller and the distribution of separations narrower. When the semi-major axis of the 
incoming binary becomes comparable to that of the BMP, the condition that the BMP not be perturbed forces a more nearly circular outer orbit for the triple (cf., $E=2.0$ ). The branching ratio for triple formation peaks at a value of about $50 \%$ corresponding to the intermediate case shown in Fig. 1b $(E=0.5)$.

When the numerical results for the triple formation cross section are combined with simple estimates for the rate at which primordial binaries drift into the cluster core by dynamical friction, one obtains the following expression for the predicted number of triple systems containing a detectable pulsar in a cluster like M4,

$$
N_{\mathrm{T}} \sim 0.1 N_{\mathrm{BMP}} \frac{f_{\mathrm{b}}}{0.1}\left(\frac{N_{\mathrm{c}}}{10^{3}}\right)^{-1}\left(\frac{N_{\mathrm{o}}}{10^{5}}\right)\left(\frac{t_{\mathrm{d}}}{10^{10} \mathrm{yr}}\right)^{-1}\left(\frac{t_{\mathrm{T}}}{10^{8} \mathrm{yr}}\right) .
$$

Here $N_{\mathrm{BMP}}$ is the number of detectable pulsars in the core, $N_{\mathrm{c}}$ is the total number of objects in the core, $N_{\mathrm{o}}$ is the number of objects in the outer region of the cluster, $f_{\mathrm{b}}$ is the binary fraction there, $t_{\mathrm{d}}$ is the dynamical friction timescale, and $t_{\mathrm{T}}$ is the lifetime of the triple in the core.

\section{Recycling Blue Stragglers to make yet more Blue Stragglers}

In the dense cores of globular clusters, a typical star has a significant chance to undergo a collision during a Hubble time. Since the velocity dispersion in globular clusters is one or two orders of magnitude less than the escape velocity at the surface of a typical main sequence star, almost all of the mass of two colliding stars is retained to form a merger product (for a detailed discussion, cf., Lombardi et al. 1995).

In a collision between relatively light stars, the merger remnant may have a mass below that of the main sequence turn-off, $0.8 \mathrm{M}_{\odot}$. In that case, it will be rather difficult to distinguish the merger product from an ordinary star of that mass. For a given candidate, detailed investigation might show unusual abundances, but spotting such stars against the background of normal stars would be akin to seeking for a needle in a haystack.

In a collision where the sum of the masses of the original stars significantly exceeds that of the turn-off, by being larger than $1 \mathrm{M}_{\odot}$ say, the merger remnant will stand out in a H-R diagram, since it will be positioned on or near the main sequence, but bluewards of the turn-off. Such a star, if born at the same time as the rest of the stars of the cluster, should have evolved away from the main sequence. Its presence thus gives the impression of having straggled, and with its relatively bluer color such a star is called a blue straggler. For a recent collection of papers on blue stragglers, see Saffer (1993).

Many, if not most, of the collisions between single stars in star clusters are likely to produce blue stragglers. One reason is that mass segregation 
will produce an excess of heavier stars in the central regions of a cluster. Other reasons are the larger radii of heavier stars, together with their enhanced gravitational focusing, both of which favor collisions for heavier, rather than lighter stars.

An even more efficient mechanism for inducing collisions is provided by binary-single-star and binary-binary encounters, specially those involving hard binaries, which have a significant chance to lead to resonant encounters where three or four stars are temporarily captured in a small area of space. Similar arguments again predict a larger collision probability for heavier stars. A special feature of 3-body or 4-body channels for blue straggler formation is that the mass of the final blue straggler may well be significantly larger than twice the turn-off mass, if more than two stars are involved in producing a single merger (cf., Davies et al. 1994).

A third way of producing collisions between two stars is through mass overflow from one component of a binary onto the other member, followed by a spiral-in, a process that does not require a high density of neighboring stars.

As a simultaneous illustration of the last two types of blue straggler formation events, a calculation by Simon Portegies Zwart is reproduced in Fig. 2 (originally exhibited as a poster during the Symposium). The calculations were performed within the Starlab environment, in which his simplified binary-evolution models were connected with the automated threebody scattering package described above.

There is nothing special about this particular set of initial conditions. Both the single star and the binary were drawn at random from a prescribed cluster core population of single stars and binaries. At each time step of a thousand years, the probabilities for close encounters between different objects were calculated, and actual collisions were carried out in Monte Carlo fashion by spinning a random number generator to see which stars would actually undergo an encounter. At each time step, those stars and binaries that did not undergo an interaction (the vast majority) were simply evolved with stellar evolution prescriptions: fitting formulas for stellar evolution tracks in the case of single stars, and simplified recipes in the case of binaries (for more details, see Portegies Zwart 1995).

In Fig. 2, an example is given of an interplay between three stars, leading to the formation of two blue stragglers. The first blue straggler is formed in isolation, as the result of binary star evolution, nearly $10 \mathrm{Gyr}$ after its formation. At this time, the $1 \mathrm{M}_{\odot}$ star attempts to climb the giant track. The first result is that its increased size leads to rapid tidal circularization, erasing the initial eccentricity of 0.3 around $T=9.97 \mathrm{Gyr}$. Soon thereafter, the primary fills its Roche lobe, and around $T=9.98 \mathrm{Gyr}$ dumps most of its mass on the secondary. 


\begin{tabular}{|c|c|c|c|c|c|}
\hline$T($ Myr $)$ & $P_{b}(d)$ & ecc & $\lambda_{1}\left(M_{\odot}\right)$ & $M_{2}\left(M_{\odot}\right)$ & $M_{3}\left(M_{\odot}\right)$ \\
\hline 0.0 & 435.9 & 0.3 & 1.0 & 0.9 & 0.8 \\
\hline 9970.0 & 379.6 & 0.0 & 1.0 & 0.9 & 0.8 \\
\hline 9975.0 & 379.6 & 0.0 & 1.0 & 0.9 & 0.8 \\
\hline 9990.0 & 213.1 & 0.0 & 0.4 & 1.0 & 0.8 \\
\hline 12000.0 & 213.1 & 0.0 & 0.4 & 1.0 & 0.8 \\
\hline 12000.0 & 174.6 & 0.5 & 0.8 & 1.0 & 0.4 \\
\hline 13445.0 & 122.8 & 0.0 & 0.8 & 1.0 & 0.4 \\
\hline 13460.0 & 122.8 & 0.0 & 0.8 & 1.0 & 0.4 \\
\hline 13464.0 & 62.9 & 0.0 & 1.0 & 0.4 & 0.4 \\
\hline 17838.0 & 62.7 & 0.0 & 1.0 & 0.4 & 0.4 \\
\hline
\end{tabular}

Figure 2. Effect of an exchange reaction on the stellar evolution of a primordial binary, with masses $M_{1}$ and $M_{2}$, encountering a single star with mass $M_{3}$, after having evolved in isolation for $12 \mathrm{Gyr}$. The orbits of the star during the reaction are plotted in the middle of the figure, with time going from left to right. The total duration of the encounter is about a hundred years. The horizontal time axis for the stellar dynamics event is therefore five orders of magnitude enlarged compared to the vertical time axis displaying the various stellar evolution events.

Each horizontal line applies to a certain age $T$ of the star cluster from which these stars are drawn. This age is given in the first column, in units of $10^{6} \mathrm{yr}$. The next three columns give the orbital period $P_{\mathrm{b}}$ of the binary, in days; the eccentricity ecc of the orbit; and the mass $M_{1}$ of the primary. The following column shows a picture of the stars within their Roche lobes, all drawn using the same scale. The next two columns give the mass $M_{2}$ of the secondary; and the mass $M_{3}$ of the single star. The remaining dot just to the right of the last column indicates the isolated nature of the third star. 
However, only $0.1 \mathrm{M}_{\odot}$ of this supply of matter can be accreted onto the companion, and $0.5 \mathrm{M}_{\odot}$ leaves the system. The reason for the inefficiency of mass transfer is that the donor star provides mass on its own thermal time scale, while the other star in turn can only accept mass on its thermal time scale, which is longer. By $T=9.99 \mathrm{Gyr}$, the primary turns into a lowmass helium-star which then cools to become a white dwarf. From this time on, the secondary shows up as a blue straggler. But before the secondary can reach the turn-off, a third star happens to pass through the system, 2 Gyr later, and is captured into the three-body resonance scattering event, displayed with time moving horizontally from left to right, for a duration of about a hundred years.

The outcome of the event, not surprisingly, leads to the lightest star being ejected, in this case the white dwarf, the end product of the original primary. The incoming $0.8 \mathrm{M}_{\odot}$ star now takes the place of the primary. The orbit has tightened significantly, as could also be expected (hard binaries tend to get harder; Heggie 1975, Hut 1983). After another $2.4 \mathrm{Gyr}$, the original secondary begins to climb the giant track, leading to a second phase of tidal circularization, in which the eccentricity induced in the three-body encounter is erased. After another $15 \mathrm{Myr}$, mass overflow takes place, again $0.6 \mathrm{M}_{\odot}$ is lost, of which this time $0.2 \mathrm{M}_{\odot}$ is accepted by exchanged star. This increases the mass of the latter from $0.8 \mathrm{M}_{\odot}$ to $1.0 \mathrm{M}_{\odot}$, thereby turning this star into a second blue straggler. The original secondary soon turns into a dwarf, orbiting the $1 \mathrm{M}_{\odot}$ star. This second straggler phase finally ends around $T=17.8 \mathrm{Gyr}$, during the last phase of giant evolution.

The final state is not displayed in this figure. After the second blue straggler will fill its Roche lobe in turn, the binary will shrink significantly, and most likely a common envelope system will be formed. This is likely to lead to the merger of the two cores, resulting in a late type giant of $1.3 \mathrm{M}_{\odot}$. A bit later, a white dwarf will then be left behind, with a mass of around $0.7 \mathrm{M}_{\odot}$.

Observationally, the first blue straggler formed by the transfer of mass in a close binary will not be distinguishable from the classical ones. Only after the dynamical exchange interaction, the system becomes clearly different from ordinary blue stragglers: the observation of a blue straggler in an eccentric orbit or in a detached binary with a main-sequence companion is a direct indication for formation by an exchange reaction.

Acknowledgements. I thank Fred Rasio and Simon Portegies Zwart for producing Figures 1 and 2, respectively, and for comments on the manuscript. 


\section{References}

Backer, D.C., Foster, R.S. \& Sallmen, S. 1993, Nat 365, 817

Davies, M.B., Benz, W. \& Hills, J.G. 1994, ApJ 424, 870

Goodman, J. 1992, in Structure and Dynamics of Globular Clusters, G. Meylan \& S. Djorgovski (Eds.), ASP Conf. Proc. Vol. 50, p. 87

Heggie, D.C. 1975, MNRAS 173, 729

Heggie, D.C. 1992, Nat 359, 772

Heggie, D.C. \& Hut, P. 1993, ApJS 85, 347

Heggie, D.C., Hut, P. \& McMillan, S. 1994, (in preparation)

Hills, J.G. 1975, AJ 80, 809

Hills, J.G. 1992, AJ 103, 1955

Hut, P. 1983, ApJ 272, L29

Hut, P. 1992, in X-ray Binaries and Recycled Pulsars, E.P.J. van den Heuvel \& S.A. Rappaport (Eds.), Kluwer Academic Publishers, p. 317

Hut, P. 1993a, ApJ 403, 256

Hut, P. 1993b, in Blue Stragglers, R.A. Saffer (Ed.), ASP Conf. Proc. Vol. 53, p. 44

Hut, P. \& Bahcall, J.N. 1983, ApJ 268, 319

Hut, P. \& McMillan, S. 1994, (in preparation)

Hut, P. et al. 1992, PASP 104, 981

Hut, P., Makino, J. \& McMillan, S. 1994, (preprint)

Lombardi, J., Rasio, F.A., \& Shapiro, S.L. 1995, ApJ (submitted)

Mikkola, S. 1984, MNRAS 208, 75

Pols, O. \& Marinus, M. 1993, in Blue Stragglers, R.A. Saffer (Ed.), ASP Conf. Proc. Vol. 53, p. 126

Portegies Zwart, S.F. 1995, (in preparation)

Rasio, F.A., McMillan, S. \& Hut, P. 1995, ApJ (in press)

Saffer, R.A. (Ed.) 1993, Blue Stragglers, ASP Conf. Proc. Vol. 53

Sigurdsson, S. \& Phinney, E.S. 1993, ApJ 415, 631

Thorsett, S.E., Arzoumanian, Z. \& Taylor, J.H. 1993, ApJ 412, L33 